Rakenteiden Mekaniikka (Journal of Structural Mechanics)

Vol. 50, No 3, 2017, pp. 158-161

https://rakenteidenmekaniikka.journal.fi/index

https://doi.org/10.23998/rm.64920

(c)The Authors 2017.

Open access under CC BY-SA 4.0 license.

\title{
Two-scale Reissner-Mindlin plate model
}

\author{
Jouni Freund ${ }^{1}$
}

Summary. A two-scale plate model, in which the displacement assumption consists of the Reissner-Mindlin and warping parts, is presented. To reduce the modelling error of the classical Reissner-Mindlin model, the warping part is chosen so that the overall displacement satisfies the full 3D elasticity equations as well as possible. Pressure loaded isotropic homogeneous plate is used as an application example.

Key words: Reissner-Mindlin, refined plate model, shear correction factor

Received 15 June 2017. Accepted 24 July 2017. Published online 21 August 2017.

\section{Introduction}

The classical Kirchhoff and Reissner-Mindlin plate models assume that the normal line segments to the midplane move as rigid bodies in deformation, and the transverse normal stress is negligible. These assumptions are the key to a simple and practical plate model but they are also the source for modelling error. In particularly, prediction of the transverse stress components is poor.

The number of different refined plate models in literature is impressive [2,3]. Increasing the polynomial order in the displacement assumption in the transverse direction is a popular choice [4,5]. The layer wise displacement assumption in [1] is another common choice for a layered plate material. One of the challenges in refinements comes from the need to compromise the modelling error with simplicity of practical calculations [5]. From this perspective, equivalent single layer theories are attractive as they keep the classical mathematical form of the plate equations.

Use of a displacement assumption which consists of the classical and warping parts is one of the ways to reduce the modelling error. In the present computational homogenization application, the RVE (Representative Volume Element) is the normal line segment to the midplane and the classical and warping displacement parts represent the slowly and rapidly varying parts of displacement in the scale of the RVE.

\footnotetext{
${ }^{1}$ Corresponding author. jouni.freund@aalto.fi
} 
The standard solution methods and software for the Reissner-Mindlin equations suffice, as the two-scale plate equations to be solved numerically are of the classical form. There, the main ingredients are the shear correction factors as predicted by the two-scale model.

\section{Two-scale plate model}

In what follows, the domain occupied by a plate is denoted by $(x, y, z) \in \Omega \times Z \subset \mathbb{R}^{3}$ in which $(x, y) \in \Omega \subset \mathbb{R}^{2}$ is the reference plane and $z \in Z \subset \mathbb{R}$ is the coordinate to the normal direction of the reference plane. According to the linear elasticity theory, displacement $\vec{u}$ of plate is given by the principle of stationarity of the total potential energy functional

$$
\Pi^{\mathrm{E}}(\vec{u})=\frac{1}{2} \int_{\Omega} \int_{Z} \vec{\sigma}_{\mathrm{c}}: \nabla \vec{u} d z d A-\int_{\Omega} \int_{Z} \vec{f} \cdot \vec{u} d z d A-\int_{\partial \Omega} \int_{Z} \vec{t} \cdot \vec{u} d z d s-\int_{\Omega} \sum_{\partial Z} \vec{q} \cdot \vec{u} d A
$$

which takes into account the internal force $\vec{\sigma}$, external volume force $\vec{f}$, external surface force $\vec{q}$ acting on the top and bottom surfaces, and external surface force $\vec{t}$ acting on the boundary surfaces.

Without additional assumptions, stationarity of the functional implies the generic equilibrium equations of linear elasticity. Equilibrium equations of plate models follow from the same principle with additional kinematic and kinetic assumptions. With the displacement assumptions of the classical Kirchhoff and Reissner-Mindlin models

$$
\begin{gathered}
\vec{u}^{\mathrm{K}}=\left(u-z \frac{\partial w}{\partial x}\right) \vec{i}+\left(v-z \frac{\partial w}{\partial y}\right) \vec{j}+w \vec{k}, \\
\vec{u}^{\mathrm{M}}=(u+z \theta) \vec{i}+(v-z \phi) \vec{j}+w \vec{k}
\end{gathered}
$$

respectively, the outcome is a boundary value problem on a two-dimensional domain $\Omega$ which results into great savings in computations compared to the computations with the generic equations on $\Omega \times Z$. The Reddy model displacement assumption [4,5]

$$
\vec{u}^{\mathrm{R}}=\vec{u}^{\mathrm{M}}-\frac{4 z^{3}}{3 t^{2}}\left(\frac{\partial w}{\partial x}+\theta\right) \vec{i}-\frac{4 z^{3}}{3 t^{2}}\left(\frac{\partial w}{\partial y}-\phi\right) \vec{j}
$$

is a modification of the Reissner-Mindlin model with a warping displacement part of 'a priori' form. The two-scale plate model employs the same idea, but treats the warping part, denoted by $\vec{v}$, as an unknown of the plate problem. The kinematic assumptions consist of

$$
\begin{gathered}
\vec{u}^{\mathrm{T}}=\vec{u}^{\mathrm{M}}+\vec{v}, \\
\nabla \vec{u}^{\mathrm{T}}=\nabla \vec{u}^{\mathrm{M}}+\vec{k} \frac{\partial}{\partial z} \vec{v}, \\
\int_{Z} \delta \vec{u}^{\mathrm{M}} \cdot \vec{v} d z=0 \quad \forall(x, y) \in \Omega \text { and } \forall \delta \vec{u}^{\mathrm{M}} .
\end{gathered}
$$

In the second assumption, the derivatives of $\vec{v}$ except that with respect to the transverse coordinate are considered to be negligible compared to the other terms of the displacement gradient. Orthogonality of the Reissner-Mindlin and warping parts in the 
third assumption implies uniqueness of the warping solution as rigid body motion of the normal line segments is included in the classical Reissner-Mindlin part. The two-scale model does not use any kinetic assumptions whereas the classical and Reddy models assume that $\sigma_{z z}=0$.

\section{Two-scale plate equations}

Derivation of the two-scale plate equations follows the usual lines of the classical plate models. The displacement assumption is substituted into the potential energy functional

$$
\Pi\left(\vec{u}^{\mathrm{M}}, \vec{\lambda}, \vec{v}\right)=\Pi^{\mathrm{E}}\left(\vec{u}^{\mathrm{M}}+\vec{v}\right)-\int_{\Omega} \int_{Z} \vec{\lambda} \cdot \vec{v} d z d A
$$

in which the Lagrange multiplier $\vec{\lambda}$ is of the same form as $\delta \vec{u}^{\mathrm{M}}$. Stationarity with respect to unknowns in $\vec{u}^{\mathrm{M}}$ gives the well-known Reissner-Mindlin plate equation set. Stationarity with respect to $\vec{\lambda}$ and $\vec{v}$ gives the equation set

$$
\begin{gathered}
\frac{\partial}{\partial z} \vec{\sigma}+\vec{f}+\vec{\lambda}=0 \text { in } Z \backslash I, \\
\llbracket \vec{\sigma} \rrbracket=0 \text { and } \llbracket \vec{v} \rrbracket=0 \text { on } I, \\
n_{z} \vec{\sigma}-\vec{q}=0 \text { on } \partial Z, \\
\int_{Z} \delta \vec{\lambda} \cdot \vec{v} d z=0
\end{gathered}
$$

which defines $\vec{\sigma} \equiv \vec{k} \cdot \vec{\sigma}, \vec{v}$ and $\vec{\lambda}$ uniquely. The jump conditions on the second row enforce the transverse stress $\vec{\sigma}$ and warping displacement $\vec{v}$ to be continuous on $I \subset Z$ where material properties are not continuous.

The two equation sets are connected by stress expression $\vec{\sigma}=\vec{\sigma}^{\mathrm{M}}+\vec{s}$ which is composed of the Reissner-Mindlin and warping parts. However, as a rather simple closed form solution to $\vec{s}$ exists, efficient implementation based on a modified constitutive equation of the Reissner-Mindlin model is possible. There, the main ingredients are the shear correction factors as predicted by the two-scale model.

\section{Isotropic homogeneous plate example}

Homogeneous isotropic plate of thickness $t$ loaded by pressure $p$ on the top surface is used as an application example. Reference plane is chosen to coincide with the midplane. Solutions to the warping displacement components

$$
\begin{aligned}
& u^{\mathrm{T}}=u^{\mathrm{M}}+\frac{1}{12} z\left(3-20 \frac{z^{2}}{t^{2}}\right)\left(\frac{\partial w}{\partial x}+\theta\right), \\
& v^{\mathrm{T}}=v^{\mathrm{M}}+\frac{1}{12} z\left(3-20 \frac{z^{2}}{t^{2}}\right)\left(\frac{\partial w}{\partial y}-\phi\right), \\
& w^{\mathrm{T}}=w^{\mathrm{M}}-\frac{v}{1-v}\left[z\left(\frac{\partial u}{\partial x}+\frac{\partial v}{\partial y}\right)+\frac{12 z^{2}-t^{2}}{24}\left(\frac{\partial \theta}{\partial x}-\frac{\partial \phi}{\partial y}\right)\right]+\frac{1-v-2 v^{2}}{1-v} \frac{t^{2}-12 t z-12 z^{2}}{24 t} \frac{p}{E}
\end{aligned}
$$


differ from the 'a priori' expressions in eq. (4). It is noteworthy that the transverse displacement of two-scale model contains a modification due to the Poisson effect. Transverse stress components

$$
\begin{gathered}
\sigma_{x z}^{\mathrm{T}}=G \frac{5}{4}\left(1-4 \frac{z^{2}}{t^{2}}\right)\left(\frac{\partial w}{\partial x}+\theta\right), \\
\sigma_{y z}^{\mathrm{T}}=G \frac{5}{4}\left(1-4 \frac{z^{2}}{t^{2}}\right)\left(\frac{\partial w}{\partial y}-\phi\right), \\
\sigma_{z z}^{\mathrm{T}}=-p \frac{t+2 z}{2 t}
\end{gathered}
$$

satisfy the traction conditions at the bottom and top surfaces $z= \pm t / 2$. The remaining stress components are given by

$$
\begin{gathered}
\sigma_{x x}^{\mathrm{T}}=\sigma_{x x}^{\mathrm{M}}+\frac{v}{1-v} \sigma_{z z}^{\mathrm{T}}, \\
\sigma_{y y}^{\mathrm{T}}=\sigma_{y y}^{\mathrm{M}}+\frac{v}{1-v} \sigma_{z z}^{\mathrm{T}}, \\
\sigma_{x y}^{\mathrm{T}}=\sigma_{x y}^{\mathrm{M}} .
\end{gathered}
$$

Integration of the transverse shear stress components over the thickness gives the classical values 5/6 of the Reissner-Mindlin model shear correction factors.

\section{References}

[1] S. Brischetto, E. Carrera, L. Demasi. Improved bending analysis of sandwich plates using a zig-zag function. Composite Structures, 89:408-415, 2009. https://doi.org/10.1016/j.compstruct.2008.09.001

[2] E. Carrera, S. Brischetto. A survey with numerical assessment of classical and refined theories for the analysis of sandwich plates. Applied Mechanics Reviews, 62:1-17, 2009. https://doi.org/10.1115/1.3013824

[3] R. Khandan, S. Noroozi, P. Sewell, J. Vinney. The development of laminated composite plate theories: a review. J Mater Sci 47:5901-5910, 2012. https://doi.org/10.1007/s10853012-6329-y

[4] M. Levinson. An accurate simple theory of the statics and dynamics of elastic plates, Mechanics Research Communications, 7:343-350, 1980. https://doi.org/10.1016/00936413(80)90049-X

[5] J.N. Reddy. A simple higher-order theory for laminated composite plates. J Appl Mech, 51(4):745-52, 1984. https://doi.org/10.1115/1.3167719

Jouni Freund

Aalto University, Department of Mechanical Engineering

Puumiehenkuja 5 A, PL 14300, 00076 AALTO

jouni.freund@aalto.fi 